\title{
BMJ Open Epidemiology of overweight and obesity in early childhood in the Gulf Cooperation Council countries: a systematic review and meta- analysis protocol
}

\author{
Mahmoud A Nahhas, ${ }^{1}$ Felix Asamoah, ${ }^{2,3}$ Stephen Mullen, ${ }^{4}$ Bright I Nwaru, ${ }^{5,6}$ \\ Ulugbek Nurmatov ${ }^{7}$
}

To cite: Nahhas MA, Asamoah F, Mullen S, et al. Epidemiology of overweight and obesity in early childhood in the Gulf Cooperation Council countries: a systematic review and metaanalysis protocol. BMJ Open 2018;8:e019363. doi:10.1136/ bmjopen-2017-019363

- Prepublication history and additional material for this paper are available online. To view these files, please visit the journal online (http://dx.doi. org/10.1136/bmjopen-2017019363).

Received 31 August 2017 Revised 16 February 2018 Accepted 19 February 2018

Check for updates

For numbered affiliations see end of article.

Correspondence to Dr Mahmoud A Nahhas; Manahhas@moh.gov.sa, mannahhas@hotmail.com

\section{ABSTRACT}

Introduction There has been a notable increase in the prevalence of overweight and obesity in school-aged children in many industrialised regions. The worldwide prevalence of childhood overweight and obesity increased from $4.2 \%$ in 1990 to $6.7 \%$ in 2010 . Although many studies have been published, the epidemiological burden of overweight and obesity in the Gulf Cooperation Council (GCC) countries (Bahrain, Kuwait, Oman, Qatar, Saudi Arabia and the United Arab Emirates) is unclear. There is a need to bring together and appraise relevant studies in order to estimate the epidemiological burden (including incidence, prevalence, risk factors, trend over time) of overweight and obesity in this region and thus help to inform national and regional policies.

Methods and analysis We will conduct a systematic review and meta-analysis on the epidemiology of overweight and obesity in early childhood including incidence, prevalence, risk factors and trends over time in the GCC countries. We will search international electronic databases including MEDLINE, EMBASE, Cochrane Library, ISI Web of Science, CINAHL, Google Scholar, AMED, Psych INFO, CAB International and WHO Global Health Library for published, unpublished and in-progress epidemiological studies of interest published from inception to 2017. In addition, we will contact an international panel of experts on the topic. There will be no restriction on the language of publication of studies. We will use the Effective Public Health Practice Project (EPHPP) to appraise the methodological quality of included studies. Meta-analysis will be undertaken using random effects models.

Ethics and dissemination Ethical approval is not required. The outcome of the review will be disseminated through conference presentations and peer-reviewed journal publication.

PROSPERO registration number CRD42017073189.

\section{INTRODUCTION}

Childhood overweight and obesity is a global health problem, impacting the physical and psychological health of individuals for the duration of their life. ${ }^{1-5}$ Childhood
Strengths and limitations of this study:

- This systematic review will for the first time provide estimates of the epidemiological burden of overweight and obesity in early childhood across the Gulf Cooperation Council (GCC) region and within each nation.

- Understanding the burden of overweight and obesity during the early years will help to formulate and strengthen national family and school policies for children in this age group in the GCC region.

- While this study will provide a comprehensive overview of the burden of overweight and obesity in the GCC region, we anticipate disparities across available studies, and this will be taken into account in our interpretation of the underlying evidence.

overweight and obesity strongly correlate with adult obesity, which is linked to an increased risk of morbidity and mortality. ${ }^{67}$ Overweight or obese children have an increased risk of heart disease, diabetes and other health problems in adulthood. ${ }^{2}$ The impact is not limited to the individual but carries a significant economic burden. ${ }^{8}$ This is partially due to increased spending on healthcare with a German study estimating the excess lifetime cost of childhood overweight and obesity in the German population at $€ 145$ billion. ${ }^{9}$

According to the WHO, 43 million children (35 million from developing countries) were estimated to be overweight and obese in 2010, while 92 million worldwide were at risk of being overweight. ${ }^{10}$ In the same report, the worldwide prevalence of childhood overweight and obesity combined increased from $4.2 \%(95 \%$ CI $3.2 \%$ to $5.2 \%$ ) in 1990 to $6.7 \%$ (95\% CI $5.6 \%$ to $7.7 \%)$ in 2010 . This trend is expected to reach $9.1 \%$ (95\% CI $7.3 \%$, $10.9 \%$ ) or affect 60 million children by $2020 .^{10}$ 
The prevalence of childhood obesity is high among Gulf Cooperation Council (GCC) countries (Bahrain, Kuwait, Oman, Qatar, Saudi Arabia and the United Arab Emirates), with the prevalence of obesity among preschool children estimated to be between $8 \%$ and $9 \% .{ }^{11}$ In Kuwait, for instance, $40 \%-49 \%$ of adolescents are obese. ${ }^{11}$ Kuwait ranks among the top $7 \%$ of countries worldwide in reference to adult obesity prevalence ${ }^{112}$ and is among the top $3 \%$ of countries with the highest prevalence of diabetes. ${ }^{13}$ The recognition of childhood overweight and obesity and the long-term consequences has made it a high priority for health authorities in the GCC countries ${ }^{1415}$ and globally. ${ }^{16}$

Currently, there is a paucity of robust epidemiological data on childhood overweight and obesity in the GCC countries. A number of studies suggest that trends in childhood overweight and obesity in GCC countries may be increasing. ${ }^{17-21}$ However, despite several studies that have been published, the epidemiological burden of overweight or obesity in the GCC is currently unclear. Given differences in population structure, culture and lifestyle habits, education, occupation, the risk factors for obesity may vary from population to population, hence risk factors may differ between the GCC countries and western countries. ${ }^{22}$ There is a need to bring together the relevant available evidence and synthesise them in order to derive relevant epidemiological parameters (including incidence, prevalence, risk factors, trend over time) that will help to describe current burden of overweight and obesity in the GCC region. This will help to formulate or strengthen existing regional and national policies around obesity, particularly in relation to school and early years nutrition and lifestyle.

The early childhood concept model and framework has been developed in several countries. ${ }^{2324}$ Obesity can occur early in life with the prenatal and early childhood periods crucial for child growth and development. ${ }^{25}{ }^{26} \mathrm{In}$ addition, important and powerful obesity risk factors can be identified within the first 1000 days of early life, from conception to 24 months. ${ }^{3}$

Preventing the causes and consequences of childhood obesity, avoiding transition of obesity from early childhood to adulthood and altering early life systems promise the interruption of the continuing vicious cycle of the childhood obesity epidemic worldwide. ${ }^{27}$

We plan to undertake a systematic review to identify, appraise and synthesise all available evidence on the epidemiology of overweight and obesity in children during the early years of life in the GCC region. Specifically, by bringing together all relevant published evidence, this systematic review aims to provide reliable and contemporaneous estimates of the incidence, prevalence, time trends, risk factors for childhood overweight and obesity in GCC countries in children during the early life years. The focus on the early years of childhood is to identify early dominant factors that may in the long-term help in formulating evidence-based policies for early interventions.

\section{METHODS AND ANALYSES}

\section{Types of studies}

We will include all interventional (randomised controlled trials, controlled before and after studies and interrupted time-series studies) and observational (cohort, casecontrol and cross-sectional studies) studies. The main source of data on the incidence and prevalence of obesity will come from cohort and cross-sectional studies, respectively. We will include interventional and case-control studies, as these will additionally provide data on risk factors for obesity. Reviews, case studies and case series and animal studies will be excluded.

\section{Participants}

The participants will include all children in their early years (up to the child's eighth birthday). We will include and extract data only for children aged under 8 years old. If there are aggregated data and no subgroup analysis for children aged up to 8 years old, we will exclude those papers.

\section{Outcome measures}

\section{Primary outcomes}

Our primary outcomes will include objectively defined measures of the incidence and prevalence of overweight and obesity, time trends in children aged up to 8 years old in GCC countries. For consistency, we will use the standard, validated WHO definition of overweight and obesity in children based on body mass index measurements.

\section{Secondary outcomes}

Secondary outcomes will include risk factors of overweight and obesity in children aged up to 8 years old in GCC countries.

\section{Search methods}

We will employ a highly sensitive search strategy to retrieve articles meeting the review criteria. We will include studies from 2000 onwards to capture current and strong evidence in this systematic review. The online Supplementary appendix 1 contains the search strategies developed for MEDLINE database interface, which we will adapt to search other databases. Databases to search will include MEDLINE, EMBASE, Cochrane Library, ISI Web of Science, CINAHL, Google Scholar, AMED, Psych INFO, CAB International and WHO Global Health Library. Additional references will be located through searching the references cited in identified papers, as well as searching databases of the proceedings of international conferences, such as ISI Conference Proceedings Citation Index and ZETOC (British Library). For unpublished and ongoing studies, we will contact international experts in the field of research. There will be no language restrictions and where possible we will translate literature in languages other than English and report any literature we are unable to translate. 


\section{Study selection}

Duplicate papers will be removed first before undertaking the screening. If there are multiple publications from the same population, we will use the main comprehensive paper that incorporates all relevant outcomes that meet our inclusion criteria. Two reviewers will independently check titles and abstracts of the retrieved articles according to the review criteria; any discrepancies will be resolved by consensus and a third reviewer will arbitrate disagreements. Full text copies of potentially relevant studies will be obtained and their eligibility for inclusion independently assessed by two reviewers; discrepancies will be resolved by consensus and disagreements will be arbitrated by a third reviewer.

\section{Quality assessment and analysis}

Two reviewers (UN and FA; MN and SM) will independently appraise the quality of eligible studies and assess the potential for risk of bias; any discrepancies will be resolved by discussion or arbitration by a third reviewer $(\mathrm{BN})$ in the event of any disagreement. We will also calculate the Kappa test a measure of the agreement between the reviewers regarding the quality of the studies. The methodological quality of intervention studies will be assessed following the recommendations of the Cochrane Effective Practice and Organisation of Care Group. ${ }^{28}$

We will assess the methodological quality of observational studies by using the Effective Public Health Practice Project (EPHPP) ${ }^{29}$ We will focus on the following domains to assess the quality of included studies: selection bias, study design, confounders, blinding, data collection method, withdrawals and dropouts and final global rating. Each component-specific parameter (ie, suitability of the study design for the research question, risk of selection bias, exposure measurement, outcome assessment and generalisability of findings) will be given a judgement: 'strong', 'moderate' and 'weak'. At the end of critical appraisal, we will also provide the overall grading for each study.

\section{Data extraction and reporting}

Two reviewers will independently extract relevant information and study data onto a customised data extraction sheet (online Supplementary appendix 2). Any discrepancies in data extraction will be resolved by discussion or arbitration by a third reviewer if agreement cannot be reached. Descriptive tables will be used to summarise the literature and characteristics of studies contributing to the overall evidence. We will employ the Preferred Reporting Items for Systematic Reviews and Meta-Analyses checklist to guide the reporting of the review. ${ }^{30}$

\section{Data analysis}

If studies deem to be reasonably clinically and methodologically homogenous, we will pool data statistically and conduct meta-analyses. Quantitative combination of the estimates will be done separately for the review outcomes: that is, separately for prevalence, incidence, risk factors and trend. Where applicable and feasible, we will convert between estimates in order to derive common estimates that will allow implementation of the meta-analysis. If the data is heterogeneous, we will undertake a narrative synthesis of the data. We will quantify the heterogeneity between studies using the $\mathrm{I}^{2}$ statistic. Where possible, we will conduct a subgroup analysis for overweight and obesity outcomes based on sex, age group, study design and country. We will perform sensitivity analyses based on risk of bias in the studies. We will assess evidence of publication bias using Funnel plots and statistically using Begg and Egger tests. ${ }^{31}{ }^{32}$ The meta-analyses will be performed using comprehensive meta-analysis, V.3.

\section{Protocol registration}

A detailed protocol for the review has been registered with the International Prospective Register of Systematic Reviews (PROSPERO CRD42017073189): http:// www.crd.york.ac.uk/prospero/ prior to commencing the review.

\section{DISCUSSION}

The current review will present the first comprehensive synthesis of the evidence relating the incidence, prevalence, time trends and risk of obesity in children in the early years up to 8 years old in GCC countries. Our preliminary searches could not find any systematic reviews on this topic.

\section{CONCLUSION}

Evidence generation and synthesis on up to date estimates of the incidence, prevalence, time trends and risk factors of obesity/overweight in children in GCC countries will help to develop interventions for overweight or obese children. Furthermore, a comprehensive synthesis of the evidence base will provide the opportunity to create evidence-informed policies in these GCC countries in the prevention of obesity and overweight among children in the early years.

\section{Author affiliations \\ ${ }^{1}$ General Administration of School Health, School Health, Ministry of Health, Kingdom of Saudi Arabia, Riyadh, Saudi Arabia \\ ${ }^{2}$ Queen Mary, University of London, London, UK \\ ${ }^{3} \mathrm{NHSI}$ England, UK \\ ${ }^{4}$ Paediatric Emergency Department, Paediatric Emergency Department, Royal Belfast Hospital for Sick Children, N.Ireland, UK \\ ${ }^{5}$ Krefting Research Centre, Institute of Medicine, University of Gothenburg, Gothenburg, Sweden \\ ${ }^{6}$ Usher Institute of Population Health Sciences and Informatics, The University of Edinburgh, Edinburgh, UK \\ ${ }^{7}$ Division of Population Medicine, School of Medicine, Cardiff University, Wales, UK}

Acknowledgements We thank the Ministry of Health of Saudi Arabia for retrieving unobtainable papers.

Collaborators Contributors: UBN and MN conceived the idea for this study. UBN, $B I N$ and FA developed the methods and together with SM and MN drafted this protocol. MN, UN, FA, SM will extract data, appraisal of papers, UN will conduct 
meta-analyses, draft a manuscript and all authors will contribute to the final synthesis of evidence.

Contributor UBN and MN conceived the idea for this study. UBN, BIN and FA developed the methods and together with SM and MN drafted this protocol. MN, UN, FA, SM will extract data, appraisal of papers, UN will conduct meta-analyses, draft a manuscript and all authors will contribute to the final synthesis of evidence.

Funding This research received no specific grant from any funding agency in the public, commercial or not-for-profit sectors.

Competing interests None declared.

Patient consent Not required.

Provenance and peer review Not commissioned; externally peer reviewed.

Open access This is an Open Access article distributed in accordance with the Creative Commons Attribution Non Commercial (CC BY-NC 4.0) license, which permits others to distribute, remix, adapt, build upon this work non-commercially, and license their derivative works on different terms, provided the original work is properly cited and the use is non-commercial. See: http://creativecommons.org/ licenses/by-nc/4.0/

(c) Article author(s) (or their employer(s) unless otherwise stated in the text of the article) 2018. All rights reserved. No commercial use is permitted unless otherwise expressly granted.

\section{REFERENCES}

1. World Health Organisation. Childhood overweight and obesity report. Report of a WHO consultation. WHO technical report series, no. 894. Geneva: World Health Organisation, 2015. http://www.who.int/ dietphysicalactivity/childhood/en/

2. Sabin MA, Kao KT, Juonala M, et al. Viewpoint article: childhood obesity - looking back over 50 years to begin to look forward. $J$ Paediatr Child Health 2015;51:82-6.

3. Mameli C, Mazzantini S, Zuccotti GV. Nutrition in the first 1000 days: the origin of childhood obesity. Int $J$ Environ Res Public Health 2016;13:838.

4. El-Bayoumy I, Shady I, Lotfy H. Prevalence of obesity among adolescents (10 to 14 years) in Kuwait. Asia Pac J Public Health 2009;21:153-9.

5. Abdulrazzaq YM, Nagelkerke N, Moussa MA. UAE population reference standard charts for body mass index and skinfold thickness, at ages 0-18 years. Int J Food Sci Nutr 2011;62:692-702.

6. Caliskan A, Atak N. A general review of childhood obesity. TAF Preventive Medicine Bulletin 2013;12:571-82.

7. Xu S, Xue Y. Pediatric obesity: causes, symptoms, prevention and treatment. Exp Ther Med 2016;11:15-20.

8. Tremmel M, Gerdtham UG, Nilsson PM, et al. Economic burden of obesity: a systematic literature review. Int J Environ Res Public Health 2017;14:435.

9. Sonntag D, Ali S, De Bock F. Lifetime indirect cost of childhood overweight and obesity: a decision analytic model. Obesity 2016;24:200-6.

10. de Onis M, Blössner M, Borghi E. Global prevalence and trends of overweight and obesity among preschool children. Am J Clin Nutr 2010;92:1257-64.

11. Ng SW, Zaghloul S, Ali HI, et al. The prevalence and trends of overweight, obesity and nutrition-related non-communicable diseases in the Arabian Gulf States. Obes Rev 2011;12:1-13.
12. WHO. "Global info base international comparisons". 2011 https:// apps.who.int/infobase/Comparisons.aspx

13. Moussa MA, Alsaeid M, Abdella N, et al. Prevalence of type 2 diabetes mellitus among Kuwaiti children and adolescents. Med Princ Pract 2008;17:270-5.

14. Ram P. Management of Healthcare in the Gulf Cooperation Council (GCC) countries with special reference to Saudi Arabia. Int J Acad Res Bus Soc Sci 2014;4:24-41.

15. Amin TT, Al-Sultan Al, Ali A. Overweight and obesity and their relation to dietary habits and socio-demographic characteristics among male primary school children in Al-Hassa, Kingdom of Saudi Arabia. Eur J Nutr 2008;47:310-8.

16. Ogden CL, Carroll MD, Kit BK, et al. Prevalence of childhood and adult obesity in the United States, 2011-2012. JAMA 2014;311:806-14.

17. Mirmiran P, Sherafat-Kazemzadeh R, Jalali-Farahani S, et al. Childhood obesity in the Middle East: a review. East Mediterr Health J 2010;16:1009-17.

18. Malik M, Bakir A. Prevalence of overweight and obesity among children in the United Arab Emirates. Obesity Reviews 2007;8:15-20.

19. Al-Sendi AM, Shetty P, Musaiger AO. Prevalence of overweight and obesity among Bahraini adolescents: a comparison between three different sets of criteria. Eur J Clin Nutr 2003;57:471-4.

20. Eapen V, Mabrouk A, Yousef S. Metabolic syndrome among the young obese in the United Arab Emirates. J Trop Pediatr 2010;56:325-8.

21. Al-Isa A, Akanji AO, Thalib L. Prevalence of the metabolic syndrome among female Kuwaiti adolescents using two different criteria. $\mathrm{Br} \mathrm{J}$ Nutr 2010;103:77.

22. Wang L, Southerland J, Wang K, et al. Ethnic differences in risk factors for obesity among adults in California, the United States. $J$ Obes 2017;2017:1-10.

23. Llywodraeth Cymru Welsh Government. Early years is one of the 5 cross cutting priorities in prosperity for all - the National strategy. http://gov.wales/topics/people-and-communities/people/childrenand-young-people/early-years/?lang=en

24. The Scottish Government. The early years framework. http://www. gov.scot/Resource/Doc/257007/0076309.pdf

25. Vine M, Hargreaves MB, Briefel RR, et al. Expanding the role of primary care in the prevention and treatment of childhood obesity: a review of clinic- and community-based recommendations and interventions. J Obes 2013;2013:1-17.

26. Pandita A, Sharma D, Pandita D, et al. Childhood obesity: prevention is better than cure. Diabetes Metab Syndr Obes 2016;9:83-9.

27. Nader PR, Huang TT, Gahagan S, et al. Next steps in obesity prevention: altering early life systems to support healthy parents, infants, and toddlers. Child Obes 2012;8:195-204.

28. Higgins JPT, eds GS. The Cochrane Collaboration. Cochrane Handbook for Systematic Reviews of Interventions Version 5.1.0. $2011 \mathrm{http}: / /$ www.cochrane.org/training/cochrane-handbook (accessed 9 Oct 2017).

29. The Effective Public Health Practice Project (EPHPP). Quality assessment tool for quantitative studies. http://www.ephpp.ca/tools. html.

30. Moher D, Liberati A, Tetzlaff J, et al. Preferred reporting items for systematic reviews and meta-Analyses: the PRISMA statement. PLoS Med 20092009;6:e1000097.

31. Begg CB, Mazumdar M. Operating characteristics of a rank correlation test for publication bias. Biometrics 1994;50:1088-101.

32. Egger M, Davey Smith G, Schneider M, et al. Bias in meta-analysis detected by a simple, graphical test. BMJ 1997;315:629-34. 